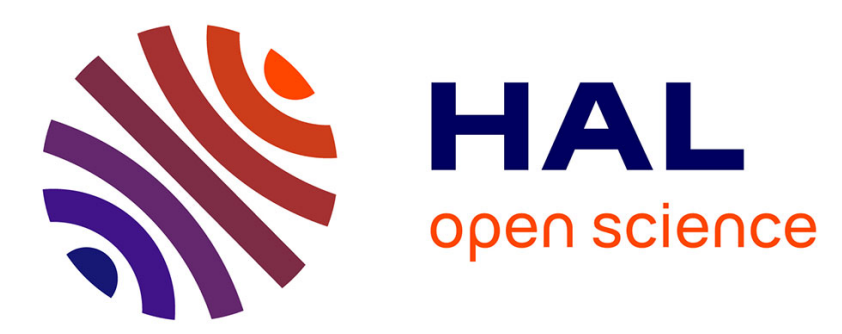

\title{
Technological Change Around The World: Evidence From Heart Attack Care
}

Abigail Moreland, Mark Mcclellan, Daniel Kessler, Olga Saynina, Michael

Hobbs, Steve Ridout, Jeff Richardson, Iain Robertson, Marie Closon, Julian

Perelman, et al.

\section{To cite this version:}

Abigail Moreland, Mark Mcclellan, Daniel Kessler, Olga Saynina, Michael Hobbs, et al.. Technological Change Around The World: Evidence From Heart Attack Care. Health Affairs, 2001, 20 (3), pp.25-42. halshs-01990623

\section{HAL Id: halshs-01990623 \\ https://shs.hal.science/halshs-01990623}

Submitted on 28 Jan 2019

HAL is a multi-disciplinary open access archive for the deposit and dissemination of scientific research documents, whether they are published or not. The documents may come from teaching and research institutions in France or abroad, or from public or private research centers.
L'archive ouverte pluridisciplinaire HAL, est destinée au dépôt et à la diffusion de documents scientifiques de niveau recherche, publiés ou non, émanant des établissements d'enseignement et de recherche français ou étrangers, des laboratoires publics ou privés. 


\section{Technological Change Around The World: Evidence From Heart Attack Care}

"Supply side" incentives to curtail health care spending are closely linked with trends in the use of costly treatments.

by the Technological Change in Health Care (TECH) Research Network

ABSTRACT: Although technological change is a hallmark of health care worldwide, relatively little evidence exists on whether changes in health care differ across the very different health care systems of developed countries. We present new comparative evidence on heart attack care in seventeen countries showing that technological change-changes in medical treatments that affect the quality and cost of care-is universal but has differed greatly around the world. Differences in treatment rates are greatest for costly medical technologies, where strict financing limits and other policies to restrict adoption of intensive technologies have been associated with divergences in medical practices over time. Countries appear to differ systematically in the time at which intensive cardiac procedures began to be widely used and in the rate of growth of the procedures. The differences appear to be related to economic and regulatory incentives of the health care systems and may have important economic and health consequences.

$\mathrm{H}$ EALTH CARE IS AN INDUSTRY that is becoming part of a global economy. Biomedical knowledge and technologies are already "global" in the sense that leading physicians in all developed countries read the same journals and electronic reviews and participate in international consortia to encourage best practices. For the most part, the same drugs and devices are available worldwide, at least within a few years of each other. Leading clinical researchers and experts collaborate internationally; leading drug and biotechnology firms think globally. As worldwide policy interest in quality of care continues to rise, international comparisons of health care systems are becoming common. Yet health care also remains a local industry, with care delivered by physicians influenced by their local peers, practice settings, and health care policies.

The Technological Change in Health Care (TECH) research net- 
work is investigating differences in technological change across countries. ${ }^{1}$ TECH is an international collaboration of investigators that is developing new international evidence on trends in treatment, resource costs, and health outcomes for common health problems. The network consists of national experts in clinical medicine, economics, and epidemiology from seventeen countries, who have developed new methods for conducting quantitative research on large administrative and clinical databases from each country. ${ }^{2}$

The TECH network focuses on the "micro" level: differences in technological change for particular types of patients. By examining particular health problems where medical treatments are known to have important effects on health, and where substantial international evidence suggests that quality of care can vary, it is potentially easier to examine the effects different health systems have on trends in quality and cost of care-that is, effects on changes in the value or productivity of health care systems. We examine trends over time rather than point-in-time differences, for two reasons. First, relatively little evidence exists on differences in changes in medical practice, even though innovation is a critical element of health care. Second, comparing trends allows us to "factor out" the genetic, cultural, social, and other influences on the incidence and treatment of diseases across countries at a point in time. These underlying factors are unlikely to change nearly as rapidly as medical practices do.

Our expectation, based on cross-sectional studies, anecdotal evidence, and previous studies, was that technological change differed greatly across countries. Determining the magnitude of such differences in trends, their association with national health system incentives, and their consequences for medical spending and health outcomes is the principal goal of our collaborative research.

Our initial focus has been on patients hospitalized with heart attacks (acute myocardial infarction, or AMI), for several reasons. Heart attack is a well-defined clinical condition around the world. Inpatient data, which are the most reliable data in most countries, are relatively complete sources of information on acute care for heart attacks. Knowledge of effective heart attack treatments has changed much in recent years; clinical trials and other data from the United States and other countries suggest that changes in medical practices may account for a large part of the improvements in outcomes. ${ }^{3}$ Thus, if differences in technological change exist across countries, they are likely to show up in inpatient care for heart attacks.

\section{Overview Of TECH Methods}

Our methods reflect efforts by the TECH research teams to identify the best available data on trends in practices in their country and to 
analyze the data consistently. To the extent possible, teams used nationally representative micro-level data sources-covering at least a large geographic area of each country-rather than reports from particular, possibly nonrepresentative institutions. ${ }^{4}$ We have developed and applied consistent methods for conducting microlevel analyses, including standardized cohort and variable definitions and population weights. More detailed presentations of the methods used to develop these data, and of these and other results from the TECH network, can be found in our other papers. ${ }^{5}$

We divide trends in technological change into two broad categories: intensive technologies (the "high-tech" procedures or other processes of care that require much expert input and resource cost each time they are used) and nonintensive technologies (processes of care that have low incremental costs). Major surgical procedures are intensive; generic drug prescriptions are nonintensive (if little careful monitoring for appropriateness, side effects, or complications is required). Many treatments fall between these extremes.

We find a great deal of technological change in most dimensions of acute heart attack care in virtually all of the countries included in our analysis. However, technological change for heart attack care has differed in many ways across countries. The results illustrate some clear relationships to health system characteristics, particularly for the case of intensive treatments for heart attack patients.

\section{Trends In Intensive Procedure Use}

We first summarize trends for countries that are able to construct longitudinal data on patient care-that is, care spanning multiple hospitalizations that is linkable to other types of records. We then turn to nonlongitudinal data, based on individual hospital discharges, which provide some evidence on the generalizability of our results to additional countries. Because almost all of our estimates are based on thousands of patients per year, standard errors of our estimates are very small. ${ }^{6}$ All of our results have been standardized for age and sex using the 1995 U.S. population of heart attack patients.

- Longitudinal data. Exhibit 1 presents standardized trends in the use of cardiac catheterization in the year after a heart attack occurs for patients hospitalized with heart attacks in six countries having longitudinal data for this treatment. Cardiac catheterization is a specialized procedure to view the blood flow to the heart; although it is diagnostic, it is a key step on the way to performing intensive procedures to improve blood flow. One obvious conclusion is that heart attack treatment has become much more intensive around the world; catheterization use has risen in all countries.

Yet technological change has occurred quite differently across 


\section{EXHIBIT 1}

\section{Trends In Cardiac Catheterization Within One Year After Heart Attack, In Five} Countries, 1989-1998

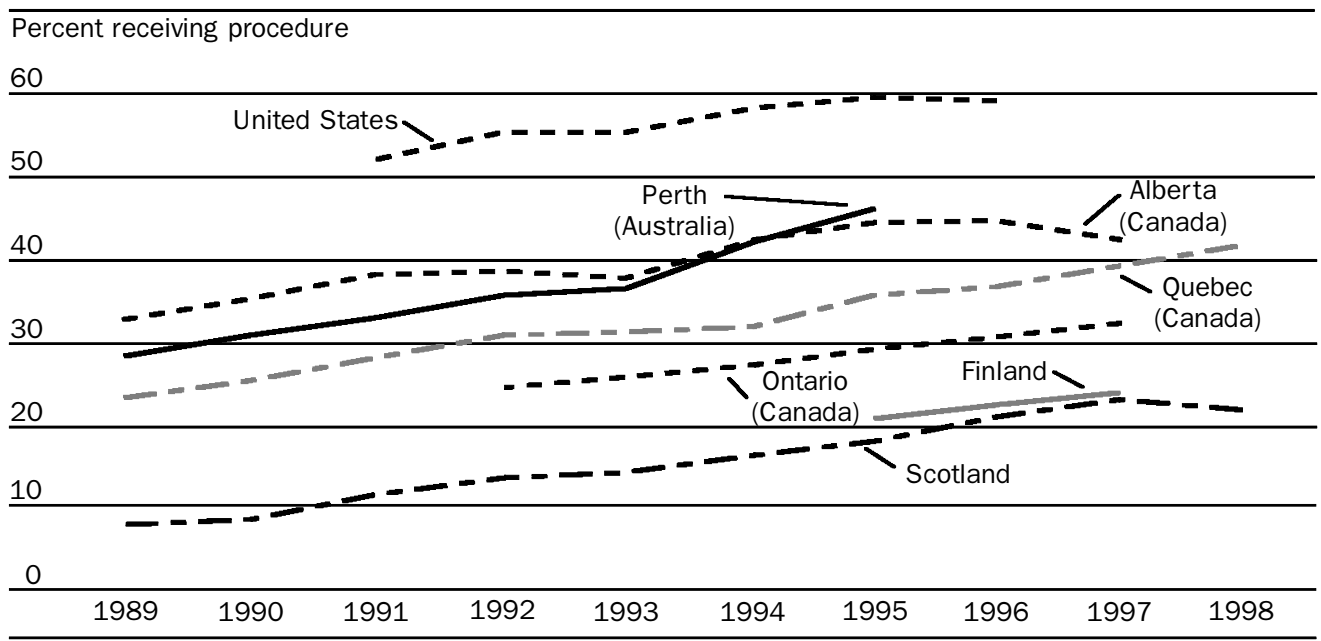

SOURCE: Authors' analysis of heart attack patient data for participating countries.

NOTES: Age- and sex-adjusted trends in the use of cardiac catheterization. The 1995 American heart attack population is used for adjustment. Percentages represent the share of all patients hospitalized with new heart attacks in the year undergoing the procedure. tern, evident in the United States, involves an early start and a fast rise. ${ }^{7}$ U.S. treatment rates are much higher than those in all of the other countries included in our longitudinal analysis.

Canada and Australia illustrate a second broad pattern of technological change for intensive treatments: later start, relatively fast growth. These countries began to use intensive cardiac procedures such as catheterization somewhat later than was the case in the United States. Later initial adoption is the primary reason that catheterization rates in Australia differ from U.S. rates. Indeed, the more rapid rate of growth in procedure use suggests that Australian practices are converging toward those in the United States. Our subsequent results also show convergence in other treatment rates for some other countries in this group.

While a number of cross-sectional studies have documented differences in catheterization rates between the United States and Canada, little evidence has existed on the differences in trends between the two countries and among the Canadian provinces. In general, Canadian catheterization rates steadily diverged from U.S. rates through 1995, before the U.S. rate leveled off. Possibly reflecting the differences in health policies across Canadian provinces, however, trends have differed greatly within Canada. For example, in association with reimbursement restrictions adopted in 1995, Alberta (which historically had relatively intensive treatment pat- 
terns) has experienced a slowdown in its growth rate in recent years. In contrast, the provincial government in Ontario, where procedure rates have been relatively low, is considering recommendations to increase procedure rates in the next few years to address the gap between it and the other Canadian provinces.

The third broad pattern of technological change is illustrated by Finland: later start and slow growth. Both the rate of use in the early years and rate of growth in use in Finland are much lower than in other countries. Thus, Finland has had much lower rates of intensive procedure use than Canada, whose use in turn has been much lower than in the United States and a few other countries. As some of our subsequent results illustrate, this pattern applies to most of the other Nordic countries and to the United Kingdom as well.

Our detailed analyses have shown that intensive treatment for the oldest heart attack patients contributes to the observed differences. At one extreme, elderly heart attack patients (age sixty-five and older) in the United States show only modest differences in treatment rates compared with nonelderly patients. In contrast, catheterization rates for the elderly compared to the nonelderly are only around three-fourths as large in Alberta (the highest-rate Canadian province) and two-thirds as large in Ontario (the lowest-rate Canadian province). And in Finland and Scotland, which have among the lowest levels and trends in procedure growth, the catheterization rate in elderly heart attack patients is only about half as high as in the overall population. In other words, there is a clear association between the absolute intensity of treatment of the population and the relative differences in the intensity of treatment between younger and older persons within the population. This is the result of relatively larger differences in trends in procedure use for elderly heart attack sufferers in different countries.

A larger number of countries were able to provide reliable data on coronary artery bypass graft (CABG) use (Exhibit 2). The same three patterns are generally evident here, as they are for the use of percutaneous transluminal coronary angioplasty (PTCA), a less invasive procedure for improving blood flow in a blocked artery. CABG rates in Israel are more similar to those of countries with the second change pattern. One reason is that PTCA rates in Israel have grown even more rapidly than they have in the United States. This difference may be attributable jointly to the relatively large supply of cardiologists and their greater influence on patient management relative to cardiac surgeons in Israel. ${ }^{8}$

On the lower end, Denmark and Scotland have had lower rates of procedure use, although growth in Denmark has been relatively rapid since the early 1990s in association with a "heart programme" 


\section{EXHIBIT 2}

\section{Trends In Bypass Surgery Within One Year After Heart Attack, In Eight Countries, 1989-1998}

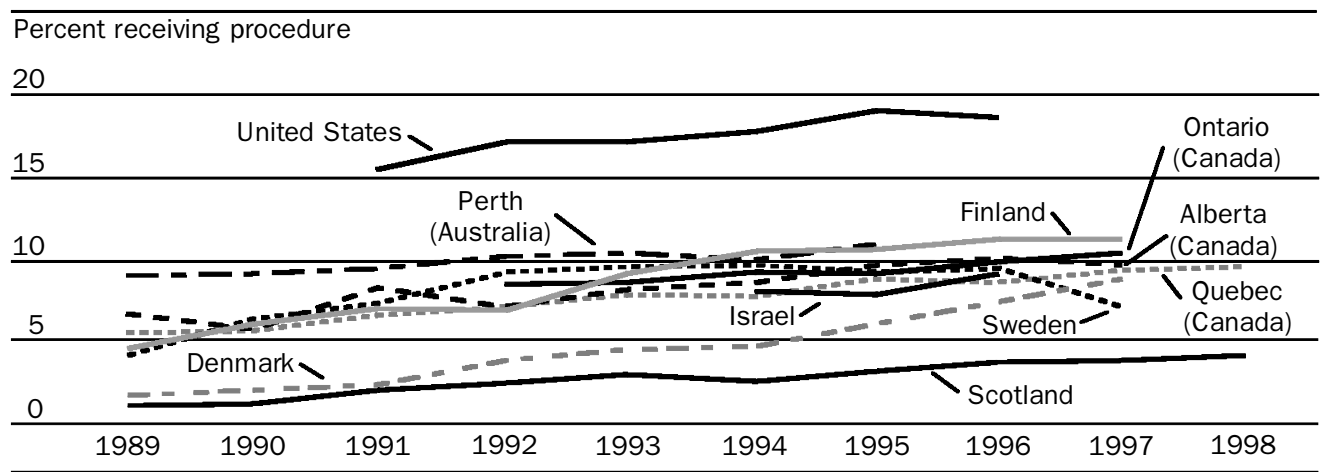

SOURCE: Authors' analysis of heart attack patient data for participating countries.

NOTES: Adjusted trends in the use of bypass (open-heart) surgery. See Exhibit 1 Notes for additional details.

explicitly intended to improve cardiac care by making intensive services more widely available. The United Kingdom has not supported any such expansions of cardiac surgery capacity, and Scotland has persistently low intensive procedure rates. Differences in angioplasty trends are even larger across countries.

Our analysis also included some recently diffusing intensive tech-

ATTACK

CARE

nologies. For example, Exhibit 3 shows rates of use of primary angioplasty - that is, PTCA performed within one day of a heart attack's occurrence, with the intention of heading off heart damage by restoring blood flow before the affected heart muscle dies. Pri-

\section{EXHIBIT 3}

Trends In One-Day (Primary) Angioplasty In Heart Attack Patients, In Five Countries, 1989-1998

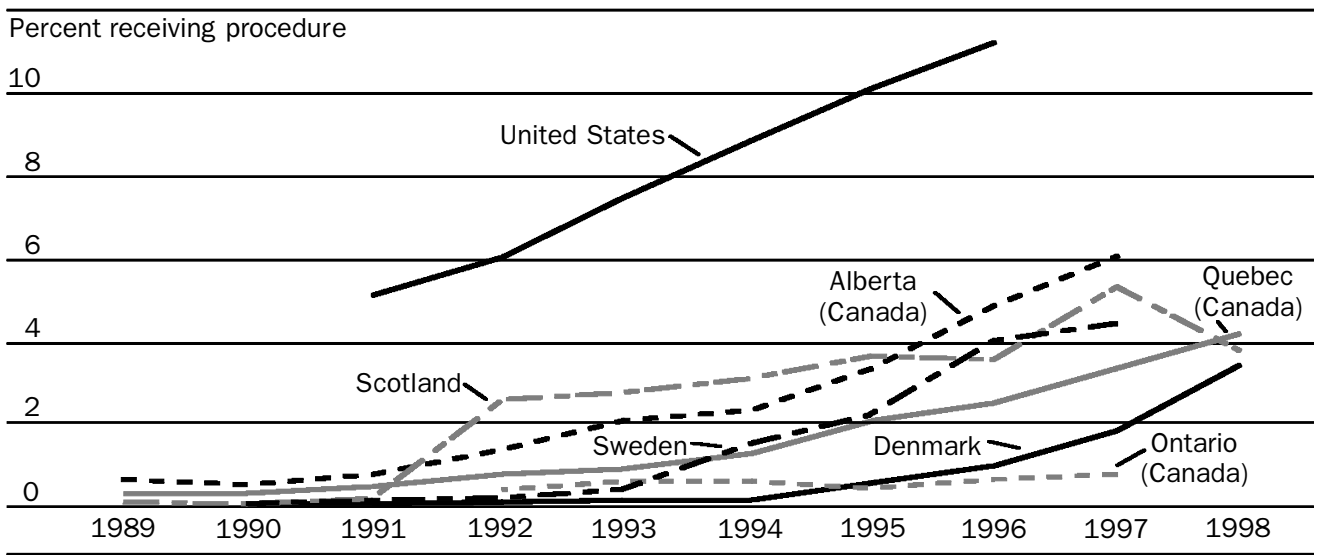

SOURCE: Authors' analysis of heart attack patient data for participating countries.

NOTES: Adjusted trends in the use of primary angioplasty (defined as angioplasty performed within one day). See Exhibit 1 Notes for additional details.

H E A L T H A F F A I R Volume $20, \mathrm{Number} 3$ 
mary angioplasty requires a rapidly available interventional cardiology team. It is a more intensive alternative to the use of another treatment to restore blood flow right after a heart attack: intravenous thrombolytic or "clot-busting" drugs that break down the blockage that caused the attack. Recently, several clinical trials have documented slightly better mortality outcomes in patients undergoing primary angioplasty, although most of these trials were published since the mid-1990s, at the end of the period of our study. ${ }^{9}$

Differences in the use of primary angioplasty are relatively larger than differences in other intensive procedures. According to Exhibit 3 , primary angioplasty was used earliest and diffused most rapidly in the United States. Diffusion started later, and has occurred more slowly, in other countries. In Ontario as well as the United Kingdom and Denmark, the procedure remains very rarely used.

For patients who undergo intensive treatment, the time to treatment has also varied across countries. Exhibit 4 shows the share of catheterization patients who received the procedure within thirty days after their heart attack in countries with relatively rapid and slow changes in intensive procedure use. Exhibit 5 shows the share of CABG patients who received the procedure within thirty days of their heart attack. Together, the two graphs provide a summary of

\section{EXHIBIT 4}

Percent Of Heart Attack Patients Undergoing Cardiac Catheterization Who Receive Procedure Within Thirty Days Of Heart Attack, In Five Countries, 1989-1998

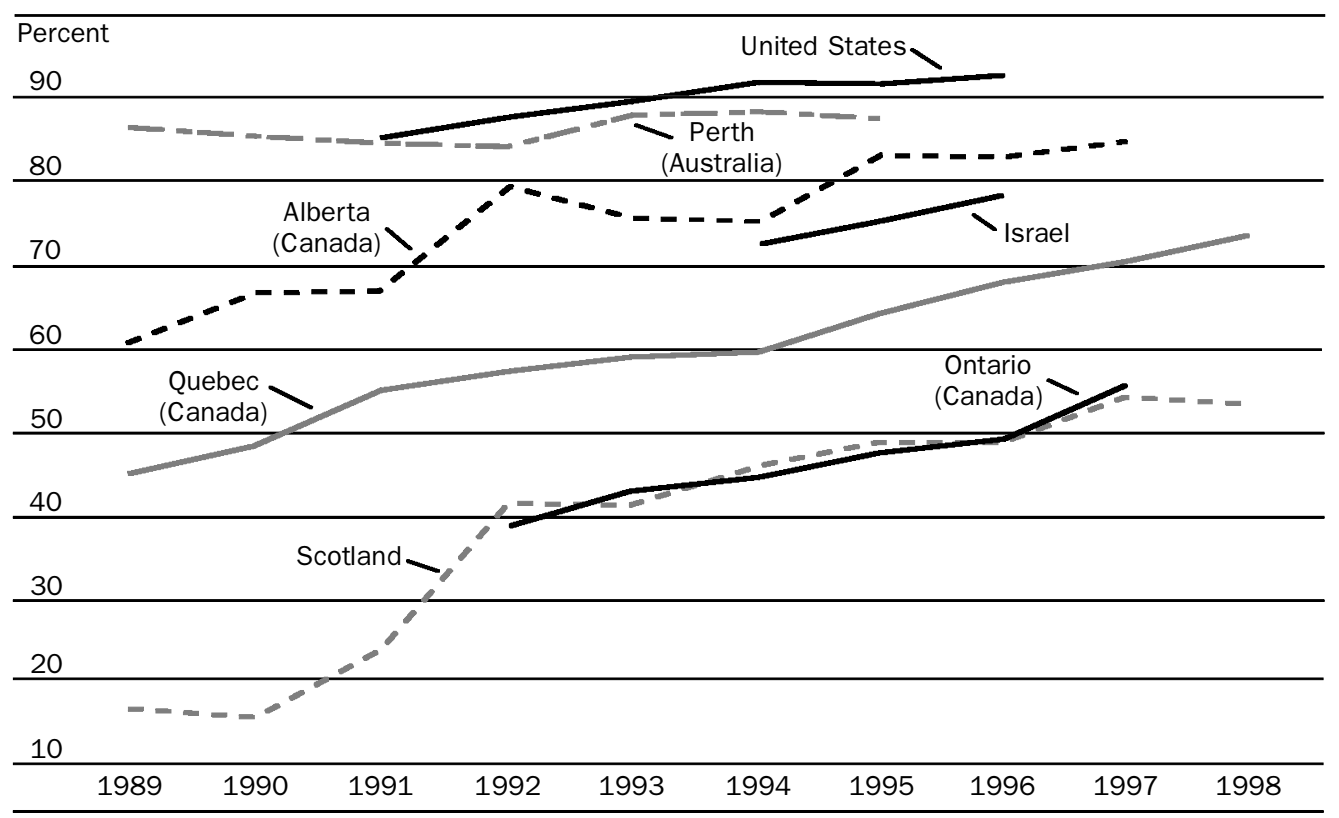

SOURCE: Authors' analysis of heart attack patient data for participating countries.

H E A L T H A F F A I R M $a y / J u n e 2001$

Downloaded from HealthAffairs.org on January 23, 2019 


\section{EXHIBIT 5}

Percent Of Heart Attack Patients Undergoing Bypass Surgery Who Receive Procedure Within Thirty Days Of Catheterization, In Five Countries, 1989-1998

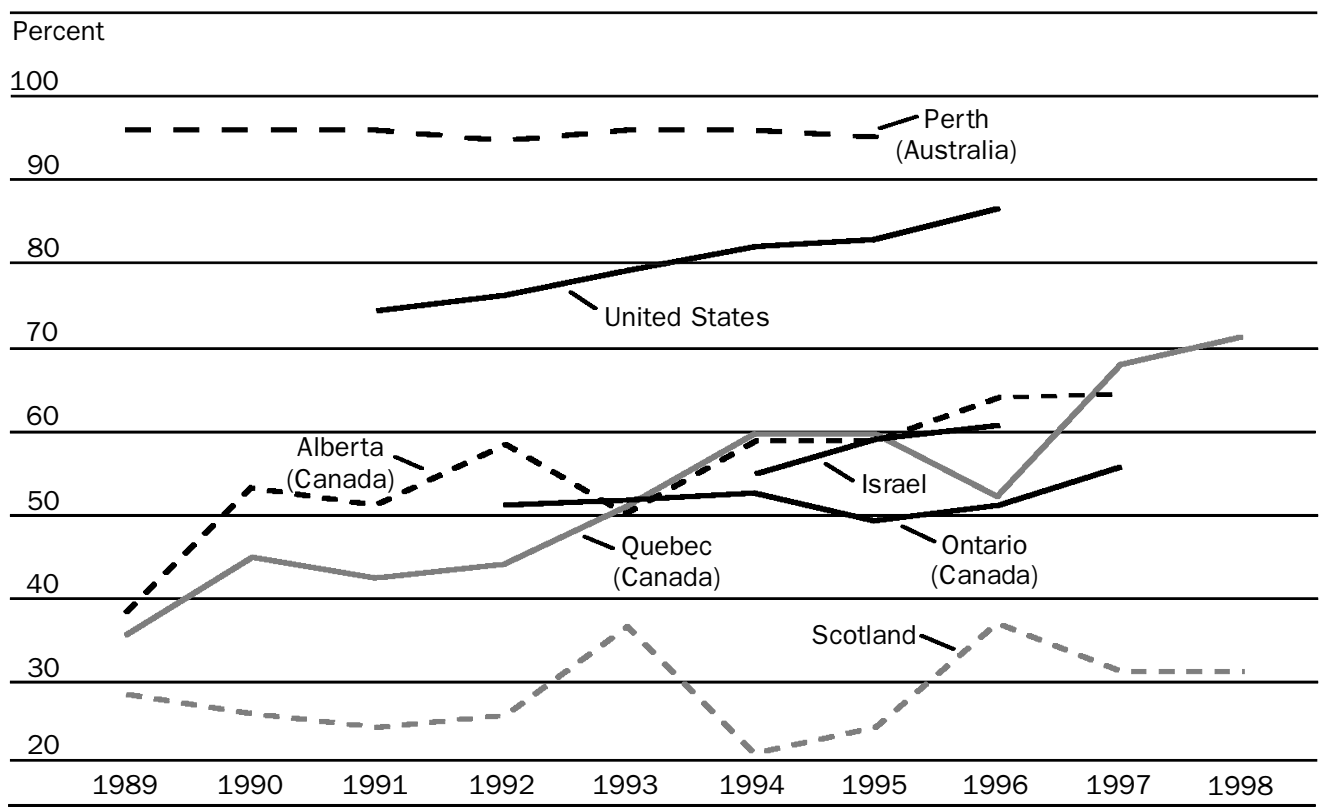

SOURCE: Authors' analysis of heart attack patient data for participating countries.

the total time to bypass surgery after heart attack.

In Australia and the United States the time to treatment is generally short: Well over 80 percent of patients undergoing catheterization do so within thirty days of the heart attack (and most of these patients do so within a week), and around 90 percent of catheterized patients who receive CABG do so within thirty days of catheterization. Alberta and Israel also have relatively rapid availability of catheterization, although the time between catheterization and CABG is somewhat greater. The other Canadian provinces have somewhat longer waiting times. For almost all of the countries and provinces, however, waits have been declining over time.

- Hospital discharge data. Nonlongitudinal discharge data on hospitalized patients are available for considerably more countries. Thus, a larger number of countries were able to estimate treatment trends within initial hospitalizations for heart attack patients. Although such trends are potentially confounded by differences in lengths of hospital stay and transfer practices, they can provide some additional evidence on technological change in intensive procedure use. Indeed, the results for countries that also performed the longitudinal analysis just described showed that differences in trends were qualitatively similar to, but more exaggerated than, the trends with longitudinal data. 


\section{EXHIBIT 6}

\section{Summary Of Trends In Catheterization Rates For Nonelderly Heart Attack Patients During Initial Hospital Admission}

\section{Pattern of technological change ${ }^{a}$}

\begin{tabular}{lll}
$\begin{array}{l}\text { Start of growth } \\
\text { in procedure use }\end{array}$ & $\begin{array}{l}\text { Growth rate after } \\
\text { use began to rise }\end{array}$ & Country \\
\hline Early & Rapid & United States, Israel \\
\hline Late & Rapid & $\begin{array}{l}\text { Australia (Victoria and Western Australia), Belgium, } \\
\text { Canada, }{ }^{b} \text { Italy, Singapore, Switzerland, Taiwan }\end{array}$ \\
\hline Unclear $^{\mathrm{c}}$ & Rapid & France, Japan \\
\hline Late & Slow & England (Oxford), Scotland, Finland, Norway \\
\hline
\end{tabular}

SOURCE: Authors' analysis of heart attack patient data for participating countries.

NOTES: Trends in catheterization rates for heart attack patients during their initial hospital stay, using nonlongitudina I data from participating countries. Catheterization rates were defined as the share of patients admitted with heart attacks undergoing catheterization during the initial hospital stay. This analysis does not require longitudinal data linked across hospitalizations, and so data are available for a larger number of countries compared to the previous exhibits.

${ }^{a}$ Rapid growth defined as an average adjusted annual growth rate of 1.5 percentage points per year or greater; slower growth is an adjusted annual rate less than 1.5 percentage points. Early adoption is defined as an observed or extrapolated (based on the years in the 1990s for which data are available) adjusted 1990 rate of ten percentage points or greater.

${ }^{\mathrm{b}}$ Growth rates in Canada (including Alberta, Ontario, and Quebec) have been somewhat slower than in Australia, Belgium, and Taiwan, especially in more recent years. But the growth rates in Alberta and Quebec are considerably higher than rates in the "slower growth" countries.

${ }^{\mathrm{c}}$ French and Japanese empirical evidence is more limited, as described in the text, so the conclusions about these countries are tentative and require further evaluation.

Exhibit 6 shows countries grouped by catheterization rates for nonelderly patients during initial heart attack admissions (that is, for admissions that were not transfers or recent readmissions) in the larger set of TECH countries able to analyze nonlongitudinal data. We summarize these results in a qualitative table because of the larger number of research teams involved, and because the qualitative presentation is less likely to be sensitive to differences in transfers and other confounding policies. ${ }^{10}$ Once again, the U.S. rate is relatively high, primarily reflecting the fact that use began to rise sharply in the 1980s, before growth began to rise in most other countries. Israel has had a pattern of growth in recent years that is similar to that of the United States.

A large set of countries have intermediate trends; growth rates are relatively similar to those in the United States, but they appear to have started to increase later, in the early 1990s. The Japanese and French trends are distinctive, in that a potentially nonrepresentative set of hospitals is included in the sample. Although the data are not sufficient to determine a "take-off date" for growth in catheterization with any precision, the growth rates in these two countries have been relatively rapid since the mid-1990s."

A third group of countries have very low rates of procedure use, growing only modestly by the end of the period. As Exhibit 1 sum-

HE A L T H A FFA R Ma 
marized, some of these countries were also able to provide longitudinal data. In these countries, catheterization rates on initial hospitalization were very low throughout the study period (much lower than Exhibit 1 would suggest). This appears to be a reflection of regionalization and referral for procedures after heart attack; patients in these countries very rarely undergo the procedure on their initial admission. In contrast, other countries are increasingly integrating the intensive procedures into the initial treatment of a heart attack.

Exhibit 7 confirms that differences in trends in intensive procedure use are important determinants of use of other relatively intensive technologies as well. For all countries able to analyze discharge data reliably, the exhibit shows trends in the use of stents, mesh tubes that have been shown to reduce the likelihood that blockage will recur after an angioplasty procedure. Stents were first introduced widely in medical device markets around 1994. As the exhibit shows, they were taken up rapidly in most countries, including those with relatively slow growth of intensive procedures. Thus, given that PTCA is performed, stent rates have grown in relatively similar ways across countries. An exception is Taiwan, where changes in reimbursement to allow for stent payments lagged.

Although discharge data are less complete than longitudinal patient records, this evidence suggests that the three major patterns of

EXHIBIT 7

Share Of Angioplasty Procedures Involving Stents In Heart Attack Admission, In Seven Countries, 1994-1998

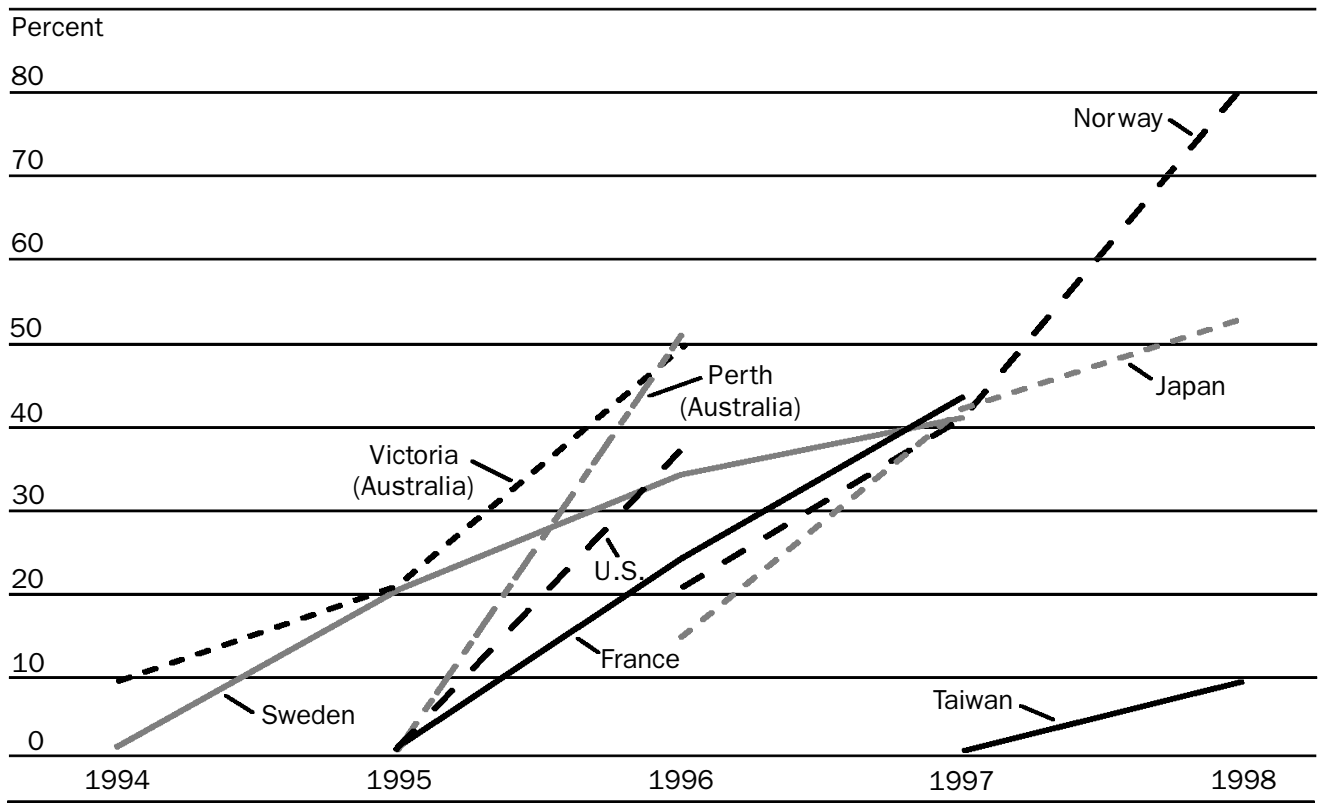

SOURCE: Authors' analysis of heart attack patient data for participating countries.

H E A L T H A F F A R - Volume 20, Number 3

Downloaded from HealthAffairs.org on January 23, 2019 
technological change involving intensive procedures evident in the longitudinal records hold for a broad range of developed countries.

\section{Trends In Less Intensive Treatments}

Few countries, including the United States, have general databases on prescription drug use linked to diagnostic and other information on large populations of patients - at least, data in a form that can be used consistently by research teams in each country. Consequently, it is not possible to develop quantitative evidence on trends in drug therapy for large, representative segments of each country's population. However, considerable qualitative evidence suggests that for the most part, trends in drug therapy have not differed as systematically across countries as have trends in intensive procedure use.

In our previous comparative work on heart attack care, our collaborating investigators found variations in technological change involving many cardiac drugs, but also some important similarities. ${ }^{12}$ For drugs that are relatively straightforward to use, such as aspirin and beta-blockers, rates of use appeared to increase greatly in virtually all participating countries. Moreover, differences in trends did not show the same systematic patterns as did intensive procedures, suggesting that the factors influencing diffusion rates for many lowcost drug treatments may be quite different than those influencing diffusion of intensive surgical treatments.

A few important differences in drug treatment trends were evident. For example, we found divergent trends in the use of thrombolytic drugs across countries. Primary angioplasty, the high-tech alternative to thrombolytics, has become an important substitute in countries such as the United States that use angioplasty most heavily. Also, trends in the choice of thrombolytic drugs have differed across countries. In the United States use of tissue plasminogen activator (tPA) has become widespread. This is a patented and thus much more costly thrombolytic, which has been shown in some trials to lead to slightly lower mortality than the generic thrombolytics (streptokinase and urokinase). ${ }^{13}$ In contrast, streptokinase is used more widely in Canada and most European countries. ${ }^{14}$

Trends in thrombolytic use rates and the choice of thrombolytics thus are correlated with trends in intensive procedure use. Overall rates of thrombolytic use have tended to increase less in countries in which intensive procedures have become more widely used; conversely, those countries are more likely to use the more costly drug when thrombolysis is performed.

A valuable source of evidence on differences in trends in drug therapy for heart attack patients is the World Health Organization's (WHO's) MONICA study, which collected clinically detailed data 
on trends in heart attack patient characteristics, acute treatments (twenty-eight days), and acute outcomes in a very large number of study sites between the mid-1980s and mid-1990s. ${ }^{15}$ While the study had some limitations, it was notable for its intensive effort to collect clinical data in a standardized format. In general, the MONICA findings confirm the conclusions of our qualitative review, in that trends in many drug therapies did not show consistent patterns and systematic differences.

For example, the MONICA investigators recently computed an "index" based on the rate of prehospital and in-hospital treatment with a set of drug therapies including beta-blockers, angiotensin converting enzyme (ACE) inhibitors, antiplatelet agents (such as aspirin), and thrombolytic drugs (in-hospital only). ${ }^{16}$ These drugs were all shown to be effective in reducing heart attack mortality before or during the period of the MONICA analysis. Overall, growth in the use of these drugs was somewhat slower in Denmark, Finland, and Sweden and somewhat faster in Australia, France, and the United Kingdom. But these patterns incorporate variable patterns across treatments: the use of certain effective drug therapies was relatively slow in some centers (for example, ACE inhibitor use in the Danish center and thrombolytic drug use in the French centers, which had more rapid diffusion of angioplasty) and relatively high in others (for example, beta-blocker use in Italy and Switzerland).

In general, however, the trends were all significantly positive and did not differ significantly from each other. Moreover, because the MONICA data only track practices through the early 1990s, they are not able to provide direct evidence on the more dramatic recent growth in many drug and surgical treatments. Thus, additional quantitative evidence on trends in the use of drug therapies in heart attack care is needed to provide a more complete comparison of technological change. However, the available evidence suggests that the differences in trends for less costly drugs are not as large or consistent across countries as the differences in procedure use are.

\section{Conclusions}

Our work illustrates that medical practices for heart attack care have changed dramatically around the world in the past decade. Treatment has become more intensive, with more use of potentially valuable medications and more use of intensive cardiac procedures. However, the way in which medical practices have changed has differed. For intensive procedures, we found three very different patterns of technological change. The United States and (based on more limited evidence) Japan and possibly France illustrated an early start/fast growth pattern: intensive procedures tended to diffuse early, 
resulting in relatively high treatment rates in the overall population in any given time period. This pattern is also associated with relatively rapid diffusion for these countries' elderly populations.

A second pattern, late start/fast growth, involves relatively rapid diffusion of intensive technologies, but diffusion that starts later and thus from a lower "base rate." These countries show diffusion rates that are similar to U.S. rates, and indeed in some cases converge toward U.S. rates. But the overall intensity of treatment at any given time tends to be somewhat lower than in the United States because of the later start of diffusion (and, in the case of Canada, because the trend rate is somewhat slower than the U.S. rate). In addition, diffusion of procedures to elderly patients in these countries tends to be slower. Countries with this pattern include Australia, Belgium, most Canadian provinces (although their growth rates were somewhat slower than those of most other countries in this group), France, Italy, Singapore, and Taiwan.

The third pattern involves late start/slow growth: later adoption and slower diffusion throughout the decade. Countries with this pattern include the United Kingdom, most of the Scandinavian countries, and (at least on some measures) Ontario.

No such systematic differences in trends were evident for relatively low-cost, easy-to-use drugs. In general, many drug treatments diffused widely in all developed countries, but the patterns of diffusion were not so clearly different. Drugs with very high costs, illustrated by tPA, showed differences in trends more like those observed for the intensive procedures.

In our ongoing work, we are conducting more comprehensive analyses of the extent to which these broad patterns of technological change are related to the underlying regulatory and economic incentives for providing medical treatments in each country. While much remains to be done, our results suggest that "supply side" incentives-particularly those affecting hospitals and, to a lesser extent, physicians-have an important relationship to observed trends in costly treatments, including intensive procedures and certain very expensive drugs. Countries such as the United States and Taiwan with relatively "weak" supply-side restrictions on the adoption of intensive treatments - such as the provision of additional reimbursement to hospitals based on the treatments they provide, and limited regulatory restrictions on particular technology adoption decisions by hospitals - have relatively rapid growth rates. Countries such as Canada, Sweden, Denmark, Finland, and Norway with stricter supply-side restrictions-such as global budgets for hospitals and central planning of the availability of intensive services-have considerably slower growth rates. The factors influenc- 
ing diffusion of drug and other therapies are somewhat less clearcut; their use is not strongly related to financial incentives. Since the costs of these treatments are relatively modest, it is possible that institutional and cultural forces as well as specific initiatives related to quality of care are primary determinants.

Policy conclusions about which of these diverse patterns of technological change are optimal depend on their consequences for patient health outcomes and costs of care in each country, and on the value placed on these outcomes by each country's population. However, it is clear that if high-quality care requires rapid innovation and diffusion of valuable high-cost as well as low-cost treatments, quality of care may differ greatly around the world, and national health policies may influence quality in important ways.

The formal evidence from clinical trials on the effects of such high-cost intensive procedures is and will likely remain limited. Especially in countries with relatively wide availability of intensive procedures, it has been difficult to find both adequate funding and adequate willingness among patients and providers to participate in randomization for such major therapeutic decisions. Moreover, because providers' experiences and use of procedures change so rapidly, the results of randomized trials may be viewed as having only limited relevance to current practice by the time they are published. This seems to have been the case in trials of primary angioplasty. The early trials in the late 1980s and early 1990s showed no benefit over thrombolytic drugs, but these trials appear to have had almost no impact on the rate of diffusion of primary angioplasty. In contrast, more recent trials have shown at least a slight advantage (one percentage point or so case survival), at least in experienced centers. Very recently, the development of complementary drugs and devices (stents) may have improved outcomes even more.

Large differences in outcome trends between countries would not be expected even if differences in procedure rates were substantial. For example, if intensive procedures convey a nontrivial mortality benefit-say, two percentage points - then even when a difference of twenty percentage points in treatment rates emerges, the associated difference in the population mortality rate would be 0.4 percentage points. Of course, this does not necessarily imply that the more intensive procedures are not worthwhile; it simply implies that careful analysis of outcome trends and the factors influencing the trends is necessary. We are conducting more detailed analyses of short-and long-term outcome trends for heart attack patients in our participating countries, and our large sample sizes provide an opportunity to detect trend differences with a very high level of precision. However, it is possible that any differences due to intensive 
procedures may be overshadowed by trend differences in less intensive treatments, population characteristics, and other factors.

Our ongoing work also suggests that more rapid diffusion of intensive technologies has had clearer implications for health care costs. If the patterns we observe for heart attack care apply more generally, then they would suggest somewhat faster medical expenditure growth in countries with the two more rapid patterns of intensive technology diffusion compared to countries with the third, slower pattern. Moreover, the material and personnel costs ("prices") associated with the use of intensive treatments also differ greatly across countries; the countries with more rapid diffusion tend to have somewhat higher payments for these inputs.

Even if the consequences for outcomes imply that the more rapid technological change involving intensive procedures is worthwhile, other important unanswered questions remain. Do the patterns we have observed for trends in acute treatments also apply to preventive services and treatments for chronic illnesses? What are the equity effects of different patterns of technological change: Does more rapid diffusion tend to exacerbate or reduce differences in utilization across socioeconomic groups, or are socioeconomic differences in use of intensive treatments unaffected? Are differences in technological change by age and by gender consequential? Does the rate of technological change affect variations in medical practice and quality of care within countries? Do countries with similar overall patterns of technological change have different outcome and cost consequences, because of differences in quality and appropriateness of treatment in patients who undergo procedures? Virtually no evidence exists on these questions. They are important next steps for international studies on how health care changes over time, and how policies can affect these changes.

This manuscript was prepared by Mark McClellan and Daniel Kessler, with assistance from Olga Saynina, Abigail Moreland, and the rest of the TECH investigators. This study was funded in part by grants from the National Institute on Aging, the Commonwealth Fund, the European Science Foundation, the Canadian Institutes for Health Research, the Australian Commonwealth Department of Health and Aged Care, the Swiss National Science Foundation (Grant nos. 3.856-0.83, 3.938-0.85, 32-9271.87, and 32-30110.90), the Swiss Heart Foundation, the Cantons of Vaud and Ticino (Switzerland), the Swedish Council for Social Research, the Swedish Medical Research Council, the Heart and Stroke Foundation of Canada, the Fonds de la Recherche en Santé du Quebec, and the Stanford University Graduate School of Business. Among others, we thank the Victoria Department of Human Services, Statistics Finland, and the Agenzia Sanitaria and the Assessorato alla Sanità of Regione Emilia Romagna for providing data. The results and

HE A L T A FFAIRS - May/June 2001

Downloaded from HealthAffairs.org on January 23, 2019 
conclusions are strictly those of the authors and should not be attributed to any of the sponsoring agencies.

\section{NOTES}

1. See M.B. McClellan and D.P. Kessler for the TECH Investigators, "A Global Analysis of Technological Change in Health Care: Preliminary Report from the TECH Research Network," Health Affairs (May/June 1999): 250-255.

2. The TECH Investigators include the following research teams. Perth, Australia: Michael Hobbs and Steve Ridout, University of Western Australia; Victoria, Australia: Jeff Richardson and Iain Robertson, Monash University Australia; Belgium: Marie Closon and Julian Perelman, Ecole de Santé Publique de l'Université Catholique de Louvain; Alberta, Canada: Konrad Fassbender, University of Alberta; Ontario, Canada: Jack Tu, Curry Grant, and Peter Austin, Institute for Clinical Evaluative Science, Toronto; Quebec, Canada: Louise Pilote and Mark J. Eisenberg, McGill University; Denmark: Terkel Christiansen and Ivar Søndbø Kristiansen, Syddansk Universitet-Odense Universitet, Mette Madsen and Søren Rasmussen, National Institute of Public Health; England: Michael Goldacre and David G.R. Yeates, Oxford University, Michael Robinson, Nuffield Institute for Health; Finland: Ilmo Keskimäki and Unto Häkkinen, National Research and Development Centre for Welfare and Health (STAKES), Veikko Salomaa and Markku Mähönen, National Public Health Institute; France: Brigitte Dormont and Carine Milcent, Université de Paris X Nanterre, Isabelle Durand-Zaleski, Hospital Henri Mondor, Santé Publique; Israel: Ethel-Sherry Gordon and Ziona Haklai, Ministry of Health, Jeremy Kark and Amir Shmueli, Hebrew University; Italy: Vincenzo Atella, University of Rome, Tor Vergata II, Daniele Fabbri, University of Bolognia, Diego Vanuzzo, Lorenza Pilotto, and Laura Pilotto, Centro Malattic Cardiovascolari, Udine; Japan: Yuichi Imanaka, Kyoto University, Yoshihiro Kaneko, National Institute of Population and Social Security Research, Haruko Noguchi, Toyo Eiwa University; Korea: Young-Hoon Kim, Korea University Medical Center, Bong-min Yang, Seoul National University; Norway: Charlotte Haug, Norwegian Patient Registry; Scotland: Alistair McGuire and Maria Raikou, City University, Frank Windmeijer, Institute for Fiscal Studies, James Boyd, Scottish Home and Health Department; Singapore: Koon Hou Mak, Kai Hong Phua, Ng Tze Pin, Ling Ling Sim, Suok-kai Chew, and Caren Tan, National Heart Centre; Sweden: Carl Hampus Lyttkens, Alexander Dozet, Anna Lindgren, Sören Höjgård, and Hans Öhlin, Lund University; Switzerland: Fred Paccaud, Bernard Burnand, and Vincent Wietlisbach, Institute of Social and Preventive Medecine (IUMSP), Alberto Holly, Lucien Gardiol, and Yves Eggli, Institute of Health Economics and Management (IEMS), University of Lausanne; Taiwan: Mei-Shu Lai, Bureau of National Health Insurance, Joan C. Lo, Institute of Economics Academia Sinica; United States: Paul Heidenreich, Daniel Kessler, Mark McClellan, Kathryn McDonald, Abigail Moreland, and Olga Saynina, Stanford University, Joseph Newhouse, Harvard University.

3. For a more detailed discussion, see E.M. Braunwald, "Shattuck Lecture-Cardiovascular Medicine at the Turn of the Millenium," New England Journal of Medicine 337, no. 19 (1998): 1360-1369; and D.M. Cutler et al., "Are Medical Prices Declining? Evidence for Heart Attack Treatments," Quarterly Journal of Economics 113, no. 4 (1998): 991-1024.

4. Most countries were able to provide national data or data from large regional databases for the analysis. U.S. data include all elderly, non-health maintenance organization (HMO) beneficiaries with new heart attacks, and all heart 
attack patients in California. Canadian data are from three provinces, as described in the text. U.K. data are from the Oxford region and Scotland. Several centers provided data from the MONICA project: Swiss data are from several prefectures surrounding Lausanne, and Italian data are from the Friuli region. Both of these samples are confined to the nonelderly. Australian data are from the states of Western Australia (Perth and surrounding areas) and Victoria. Only two countries did not have approximately representative regional or national data. French data are from all public and nonprofit private hospitals, which represent about two-thirds of heart attack stays. Japanese data are from a selected sample of six large, academically oriented hospitals. We discuss the potential nonrepresentativeness of the French and Japanese results in more detail below. All other research teams analyzed national data sets.

5. See M.B. McClellan and D.P. Kessler, eds., A Global Analysis of Technological Change in Health Care: Heart Attacks (Ann Arbor: University of Michigan Press, forthcoming). Following previous validated studies in multiple countries, all research teams used a consistent case definition for AMI patients based on discharge data, applying the same exclusions to avoid cases unlikely to represent true new AMIs. See M.B. McClellan et al., "Trends in Intensive Procedure Use and Outcomes in the United States and Canada" (forthcoming). We have also compared comorbidities and comorbidity trends across countries, and (in other work) have estimated multivariate models with and without various sets of comorbidity control variables. See J.V. Tu et al., "Development and Validation of the Ontario Acute Myocardial Infarction Mortality Prediction Rules," Journal of the American College of Cardiology 37 (2001). In general, these models show that after demographic adjustment, little to no difference exists between trend results estimated using models that account for comorbidities and those without.

6. Since essentially the whole population of many of the countries is included, standard errors are not needed for reaching conclusions about differences in the treatment trends observed in participating countries' populations.

7. In this and subsequent exhibits, results are plotted for countries only for the years in which complete and valid data could be obtained. Because the time periods of valid data differ across countries, the years of results plotted differ across countries. One consequence of this is that the earliest data shown for an "early start" country, such as the United States, may be for a year that is subsequent to the earliest data shown for some "late start" countries. The determination of "early start" status was made primarily based on a comparison of procedure rates across time. For example, in the case of catheterization, U.S. procedure rates are much higher at any given time than in virtually all other countries, so it seems reasonable to conclude that the U.S. rate started growing first. Unless otherwise noted, we focus on absolute rates of growth, not relative ones, for several reasons. First, we are often interested in rates soon after procedures start to diffuse; in this case, the denominator is close to zero, so we would get very high relative rate growth even if the absolute rate growth is negligible. Second, what matters most for changes in costs and possibly outcomes in a country is the change in share of patients getting a procedure, not the relative increase in procedures. Finally, in examining the data, many of the absolute growth rates are similar despite some differences in levels, and a relative rate comparison would obscure that point.

8. Bypass surgery rates in Israel may be underestimated, because private facilities that perform elective invasive cardiology and bypass surgery (but do not admit AMI patients) did not have data available for linkage.

9. For references to the extensive literature on these trials, contact the authors, $<$ Mark_McClellan@odp.eop.gov>.

H E A L T H A F F A R S M a y/June 2001

Downloaded from HealthAffairs.org on January 23, 2019 
10. That is, heart attack patients who undergo catheterization after transfer are not included; because a much larger share of procedures occur after such referrals in these countries, the procedure rates appear particularly low.

11. The absence of any central collection of data on treatments in Japan precludes a complete analysis of trends in intensive procedures. The Japanese sample consists of a set of six relatively large "voluntary" hospitals, which are likely to have more intensive treatment practices than many other, smaller Japanese hospitals. These hospitals show both high catheterization rates and rapid growth, but the rates for all of Japan are likely to be somewhat lower.

The French data consist of all public and private not-for-profit hospitals, which have somewhat lower rates of procedure use than private for-profit hospitals in recent years (unfortunately, no trend data are yet available for private hospitals). This higher rate may reflect the differences in reimbursement incentives between the public and private sectors (that is, the private hospitals rely on fee-for-service payments). Thus, although the observed French rates are somewhat lower than U.S. rates (with a similar rate of growth), it is likely that the French rates for the country as a whole are greater, making it difficult to reach definitive conclusions about whether catheterization rates in France lag behind those in the United States and Israel.

12. McClellan and Kessler, A Global Analysis of Technological Change in Health Care.

13. Early trials of tPA versus streptokinase detected no difference in effects. However, trials in the 1990s evaluated more rapid and sophisticated delivery protocols suggested by clinical experts and have shown a small advantage of tPA in restoring coronary blood flow and in long-term survival. In particular, the GUSTO trial observed a one-percentage-point difference in risk of short-term death that persisted at one year. More recent trials involving tPA have evaluated alternative ways and adjuvant treatments for delivering the drug, also with modest improvements in outcomes. See, for example, I.K. Jang et al., "A Multicenter, Randomized Study of Argatroban versus Heparin as Adjunct to Tissue Plasminogen Activator (TPA) in Acute Myocardial Infarction: Myocardial Infarction with Novastan and TPA (MINT) Study," Journal of the American College of Cardiology 33, no. 7 (1999): 1879-1885.

14. For example, the protocol for Montreal General Hospital states that the groups most likely to benefit from tPA should get it. In a Quebec registry of AMI collected in 1995-1996 among forty-four Quebec acute care hospitals and 3,741 patients with AMI of whom 1,357 received thrombolytic therapy, 68.2 percent received streptokinase and 31.8 percent received tPA. J. Brophy, "Falling Hospital Mortality for Acute Myocardial Infarction in Quebec Hospitals," Canadian Journal of Cardiology 14, no. 11 (1998): 1358-1362.

15. See H. Tunstall-Pedoe for WHO MONICA Project Principal Investigators, "The World Health Organization MONICA Project (Monitoring Trends and Determinants in Cardivascular Disease): A Major International Collaboration," Journal of Clinical Epidemiology 41 (1988): 105-114; and H. Tunstall-Pedoe et al., for the WHO MONICA Project, "Estimation of the Contribution of Changes in Coronary Care to Improving Survival, Event Rates, and Coronary Heart Disease Mortality across the WHO MONICA Project Populations," Lancet 355 (2000): 688-700.

16. Tunstall-Pedoe et al., "Estimation of the Contribution of Changes." 